(A) Check for updates

Cite this: Nanoscale, 2020, 12, 6959

Received 9th February 2020,

Accepted 8th March 2020

DOI: 10.1039/d0nr01138j

rsc.li/nanoscale

\section{Surface functionalization of gold nanoclusters with arginine: a trade-off between microtumor uptake and radiotherapy enhancement $\uparrow$}

\author{
Mans Broekgaarden, (iD *a Anne-Laure Bulin, (iD ${ }^{b}$ Estelle Porret, (iD) a \\ Benjamin Musnier, (D) a Benoit Chovelon, ${ }^{c, d}$ Corinne Ravelet, ${ }^{c}$ Lucie Sancey, (DD ${ }^{a}$ \\ Hélène Elleaume, (iD ${ }^{b}$ Pierre Hainaut, (iD ${ }^{a}$ Jean-Luc Coll (iD *a and \\ Xavier Le Guével iD a
}

\begin{abstract}
Ultra-small gold nanoclusters (AuNCs) are increasingly investigated for cancer imaging and radiotherapy enhancement. While finetuning the AuNC surface chemistry can optimize their pharmacokinetics, its effects on radiotherapy enhancement remain largely unexplored. This study demonstrates that optimizing the surface chemistry of AuNCs for increased tumor uptake can significantly affect its potential to augment radiotherapy outcomes.
\end{abstract}

Radiotherapy enhancement strategies are critically needed for the management of various cancer types. For glioblastoma, radiation regimens are challenging to design: high radiation doses are needed to control tumor growth, yet applicable radiation doses are limited to remain tolerable to surrounding healthy brain tissue. ${ }^{1}$ Strategies that render cancer tissues more susceptible are highly desired to make lower radiation doses more efficient, so that effective cancer management can be achieved with minimal damage to healthy tissues.

Ultra-small gold particles called nanoclusters (AuNCs) are being increasingly investigated for cancer imaging and have the potential to augment radiotherapy. ${ }^{2,3}$ AuNCs are typically composed of tens to hundreds of gold atoms corresponding to a metal core smaller than $3 \mathrm{~nm}$, which can be stabilized in solution by a variety of biomolecules such as peptides, proteins or DNA. $^{3-5}$ This flexibility in terms of core size, ligand coverage,

\footnotetext{
${ }^{a}$ Institute for Advanced Biosciences, Université Grenoble-Alpes, INSERM U1209, CNRS UMR 5309, Allée des Alpes, 38700 La Tronche, France.

E-mail: mans.broekgaarden@univ-grenoble-alpes.fr,

jean-luc.coll@univ-grenoble-alpes.fr

${ }^{b}$ Synchrotron Radiation for Biomedicine, UA 07 INSERM (STROBE), Université Grenoble-Alpes, European Synchrotron Radiation Facility, 71 avenue des Martyrs CS40220, 38043 Grenoble, France

${ }^{c}$ Université Grenoble-Alpes, Département de Pharmacochimie Moléculaire UMR 5063, 470 rue de la Chimie, 38400 St Martin d'Hères, France

${ }^{d}$ Institut de Biologie et Pathologie, CHU de Grenoble-Alpes, 9 Boulevard de la

Chantourne, 38700 La Tronche, France

$\dagger$ Electronic supplementary information (ESI) available. See DOI: 10.1039/ donr01138j
}

and functionalization can be used to tune their photoluminescence in the near-infrared (NIR, 700-900 nm) ${ }^{6}$ and shortwave infrared (SWIR, 1000-1700 nm) ${ }^{7,8}$ for in vivo imaging and optical detection of cancer, ${ }^{9}$ blood vasculatures, ${ }^{8}$ and also for fluorescence-guided surgery. ${ }^{10}$

As Au is a high $Z$-atom $\left(Z_{\mathrm{Au}}=79\right)$, it is capable of absorbing orthovoltage $(<250 \mathrm{keV}) \mathrm{X}$-rays more efficiently than water and soft tissue. ${ }^{11,12}$ By itself, radiotherapy induces DNA damage, either by direct one-electron damage to DNA bases, or by the radiolysis of water to produce highly cytotoxic hydroxyl radicals $\left({ }^{\circ} \mathrm{OH}\right) .{ }^{13}$ However, the presence of $\mathrm{Au}$ in tumor tissues during radiotherapy can result in high degrees of radiation absorption, after which the energy is locally deposited to enhance radiation-induced tissue damage by increased generation of ${ }^{\circ} \mathrm{OH} .{ }^{14,15}$ Hainfeld et al., were the first to demonstrate the augmentation of $250 \mathrm{kVp}$ radiotherapy, yielding greatly improved survival rates in syngeneic subcutaneous mouse models of breast cancer. ${ }^{16}$ Similarly, 2 to 3 -fold improved reductions in tumor weight were achieved by AuNC-augmented radiotherapy (662 keV) in mice bearing subcutaneous ovarian cancer. ${ }^{17-19}$

Our group recently demonstrated that zwitterionic sulfobetaine stabilized AuNCs have the capacity to accumulate in brain tumors. ${ }^{20}$ To further improve tumor uptake, we developed AuNCs functionalized with arginine (AuSG-2Arg), ${ }^{21}$ which demonstrated rapid accumulation in cancer cells, making them potentially interesting for radiotherapy enhancement.

This study specifically explored the radiotherapy enhancement of two AuNCs with distinct surface chemistries, namely glutathione-stabilized AuNCs (AuSG), and the novel argininestabilized AuNCs (AuSG-2Arg). The theranostic properties of these AuNCs were evaluated using 3D culture models of glioblastoma, as these more faithfully recapitulate cancer growth. We demonstrate that, despite achieving an 11-fold increase in tumor accumulation, AuSG-2Arg are less efficient at augmenting radiotherapy compared to the AuSG. Modifying the surface chemistry of AuNCs thus strongly influences their radiothera- 

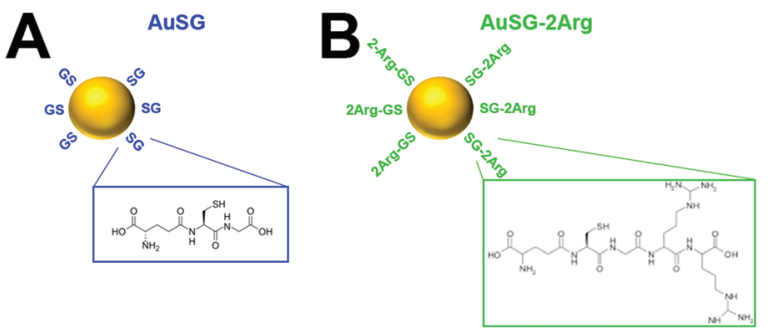

Fig. 1 Schematic overview of the AuSG and AuSG-2Arg.

peutic potential. These findings are critical to the design of novel AuNCs for imaging and cancer therapy.

AuSG and AuSG-2Arg nanoclusters (Fig. 1) were prepared by a bottom-up approach as described previously. ${ }^{21}$ Suspended spheroids were established in ultra-low-adhesion plates from human U87MG and rat F98 glioblastoma cell lines. Radiotherapy was given using monochromatic $50 \mathrm{keV}$ synchrotron radiation. Multiparametric assessment of radiotherapy outcomes was performed as described previously. $^{22}$ Assessment of necrosis was based on cell permeability using propidium iodide staining. Oxidative stress was evaluated using the dichlorodihydrofluorescein $\left(\mathrm{DCFH}_{2}-\mathrm{DA}\right)$ method. ${ }^{23} \mathrm{~A}$ detailed Materials and methods section is provided in the ESI. $\dagger$

The physicochemical characteristics of the AuNCs are reported in Fig. S1.† High resolution transmission electron microscopy of AuSG and AuSG-2Arg showed core sizes between 2 and $4 \mathrm{~nm}$ with semi-crystalline structure. The hydrodynamic diameter was $1.66 \pm 0.01 \mathrm{~nm}$ for AuSG and $2.02 \pm 0.02 \mathrm{~nm}$ for AuSG-2Arg. The zeta potentials at $\mathrm{pH} 7$ were $\zeta=-31.05 \pm$ $1.12 \mathrm{mV}$ for AuSG and $\zeta=+7.96 \pm 0.44 \mathrm{mV}$ for AuSG-2Arg as expected after addition of Arg. The AuNCs have similar absorption spectra with gradually declining intensities in the range of 400-900 nm. Fluorescence emission profiles are also comparable between the two AuNCs, with the AuSG-2Arg exhibiting an increased emission intensity at $670 \mathrm{~nm}$ (Fig. S1†).

Next, the acute $(12 \mathrm{~h})$ and prolonged ( $96 \mathrm{~h}$ ) toxicity of the AuNCs was evaluated on U87MG and F98 glioblastoma spheroids. The results depict that both AuNCs exerted minor toxicity ( $<10 \%$ reduction in viability, $5 \%$ increase in necrosis) at concentrations exceeding $100 \mu \mathrm{g}$ Au per mL, most notably in F98 spheroids (Fig. S2 $\dagger$ ). Thus, the AuNCs were generally well-tolerated and all subsequent experiments were performed using an intrinsically non-toxic dose of $100 \mu \mathrm{g}$ Au per mL. We also evaluated the AuNC toxicity on non-malignant endothelial cells and mesothelial cells, as these are the first tissues exposed to AuNCs during either intravenous or intraperitoneal administration. The results demonstrate that the particles were well tolerated by both cell types (Fig. S3†).

The uptake of the AuNCs was evaluated using inductivelycoupled plasma mass spectrometry (ICP-MS), NIR-, and SWIR fluorescence imaging. The ICP-MS results indicate that there was a significant, 4-fold (U87MG) and 13-fold (F98) increase in uptake of AuSG-2Arg as compared to AuSG (Fig. 2A, Fig. S2 and $\mathrm{S} 3 \dagger$ ). The uptake of both AuNCs typically reached a


Fig. 2 Arginine-functionalized gold nanoclusters are efficiently taken up by U87MG glioblastoma spheroids. (A and B) Quantification of AuNCs uptake by ICP-MS (A), and NIR confocal fluorescence microscopy (B). (C) NIR fluorescence images of AuNCs in U87MG spheroids following a $12 \mathrm{~h}$ incubation. AuNC fluorescence is depicted in yellow, scalebar = $200 \mu \mathrm{m}$. (D) Fluorescence intensity profiles obtained from the radius of the spheroids are shown in $C$. Data represents the mean $\pm \operatorname{SEM}(N \geq 6)$ from 3 technical repeats.

plateau after $12 \mathrm{~h}$ of incubation. Semi-quantitative NIR fluorescence imaging confirmed similar kinetics of AuNCs uptake for both spheroid types (Fig. 2B, Fig. S4 and S5†). Fluorescence intensity plots of the NIR images revealed that AuSG-2Arg accumulated predominantly in the first few cell layers of the spheroids, with fluorescence intensity values reaching background levels at approximately $100 \mu \mathrm{m}$ from the edge of the spheroid at $2 \mathrm{~h}$ and $12 \mathrm{~h}$ incubation times (Fig. 2D, Fig. S4 $\dagger$ ). In contrast, AuSG also accumulate in the first few cell layers after $12 \mathrm{~h}$ of incubation, but their fluorescence emission was also detected deeper inside the spheroids (Fig. 2D). After $24 \mathrm{~h}$ incubation, both the AuSG-2Arg and AuSG nanoclusters demonstrated peak intensities at the spheroid periphery (Fig. S4†). Similar findings were observed for the F98 spheroids (Fig. S5 $\dagger$ ). As AuNCs exhibit broad fluorescence emission in the near-infrared, it was also possible to detect their presence at longer wavelengths in the SWIR window. These confirmed a similar trend as compared to the NIR imaging for AuSG-2Arg, and to a lesser extent for AuSG (Fig. S4 and S5 $\dagger$ ). Transmission electron microscopy revealed homogeneous cytosolic and endosomal localization of both AuNCs, while the AuSG-2Arg also formed extracellular aggregates (Fig. S6†).

We next investigated the radiosensitization of the AuNCs in U87MG and F98 3D cultures of glioblastoma. The X-ray absorption of $\mathrm{Au}$ is well-characterized to peak at an energy of $81 \mathrm{keV}$ (K-edge), although the highest absorption difference with water is at approximately $50 \mathrm{keV}{ }^{11}$ Spheroids were incubated with AuSG-2Arg or AuSG (100 $\mu \mathrm{g}$ Au per mL) for $12 \mathrm{~h}$, after which free AuNCs were washed away. Immediately thereafter, 
A

the glioblastoma spheroids were exposed to different doses of $50 \mathrm{keV}$ monochromatic synchrotron radiation. In U87MG spheroids, we demonstrate a significant dose-dependent inhibition of spheroid growth (Fig. 3A and B), which was not influenced by the AuNCs. Multiparametric treatment analysis revealed that U87MG spheroid viability decreased in a radiation-dose dependent manner, and the radiotherapy efficacy was significantly improved by AuSG (1.4-fold), but not by AuSG-2Arg (Fig. S7 \& S8†). Although radiotherapy, through its induction of wide-spread DNA damage, typically associates with reduced cancer cell proliferation and apoptosis, ${ }^{24}$ we demonstrate that both AuSG and AuSG-2Arg induced a radiation-dose-dependent increase in radiotherapy-induced necrosis compared to spheroids receiving radiotherapy alone (Fig. 3C). The dose response fits based on spheroid necrosis indicated a 1.8- and 1.5-fold increase in efficacy for AuSG and AuSG-2Arg, respectively. By comparatively investigating the size and level of necrosis of each spheroid, it can be seen that radiotherapy with AuSG and AuSG-2Arg results in smaller masses with higher degrees of necrosis compared to radiotherapy alone (Fig. $\mathrm{S} 7 \dagger$ ).

In the F98 spheroids, quantification of the spheroid viabilities and levels of necrosis indicated that there was also a significant improvement in the radiotherapy efficacy following AuSG exposure (1.9-fold and 1.5-fold based on viability and necrosis, respectively), but not for AuSG-2Arg (Fig. S9 \& S10†).

These findings were surprising given the substantially elevated levels of AuSG-2Arg in the spheroids compared to AuSG. When assuming that the spheroids are spherical with a diameter of $500 \mu \mathrm{m}$, we estimated Au concentrations after a $12 \mathrm{~h}$



Ox. Stress 5 days post radiotherapy
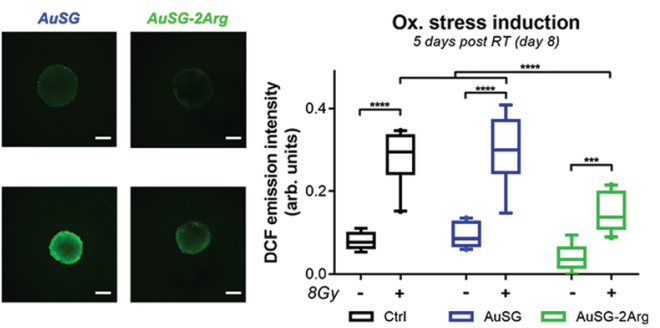

Fig. 4 Detection of intracellular ROS following radiotherapy reveals reduction in radiotherapy-induced oxidative stress by AuSG-2Arg. Fluorescence microscopy images and quantification of DCF-emission of 8 day-old U87MG spheroids incubated with $\mathrm{DCFH}_{2}$-DA following radiotherapy (5 days post-treatment). Scalebar $=200 \mu \mathrm{m}$. Data represents the mean $\pm \operatorname{SEM}(N \geq 6)$ from 2 technical repeats.

incubation in the U87MG spheroids in the range of 20-80 $\mu \mathrm{g}$ Au per $\mathrm{mL}$ for AuSG and AuSG-2Arg. Our findings are therefore unlikely to be explained by a bona-fide dose-enhancement effect, which requires far higher intratumor concentrations of gold in the order of $\mathrm{mg} \mathrm{mL} \mathrm{m}^{-1} \cdot{ }^{25}$ Our results appear best supported by the findings of Cheng et al., who reported that $\mathrm{Au}$ nanoparticles could act as chemical catalysts in the production of reactive oxygen species (ROS) during radiotherapy. ${ }^{12,26}$ Inspection of oxidative stress levels in the U87MG spheroids showed that radiotherapy induced sustained oxidative stress, as measured 5-days post-treatment. However, this was not elevated in the presence of the AuNCs. In contrast, the extent of oxidative stress in the AuSG-2Arg group was significantly lower compared to the control and AuSG groups (Fig. 4). Further investigations demonstrated that AuSG elevates basal levels of oxidative stress in both U87MG and F98 cells, whereas AuSG-2Arg was only able to do this in U87MG cells, and to a lesser extent compared to AuSG (Fig. S11C \& Fi). The findings overlap well with the radiotherapy efficacy findings in both spheroid types (Fig. $3 \& \mathrm{~S} 7 \dagger$ ). When incubated with $\mathrm{Fe}^{2+}$ to generate ${ }^{\circ} \mathrm{OH},{ }^{27}$ i.e., the same ROS produced during radiotherapy, we found significantly lower levels of oxidative stress in cells exposed to AuSG-2Arg compared to AuSG (Fig. S11D \& G).

Recent works have demonstrated that AuNCs have high potential as theranostic agents for cancer imaging and radiosensitization. Despite exhibiting promising tumor retention, ${ }^{9}$ their exploitation is challenged by their suboptimal pharmacokinetic profiles. ${ }^{10,20}$ To improve this, we and others continue to develop novel functionalized AuNCs. However, the theranostic evaluation of emerging AuNCs with 2D cultures have often produced promising results that failed to be effectively translated to in vivo studies. To better bridge the gap between in vitro and in vivo research, this study focused specifically on the use of $3 \mathrm{D}$ culture models, as these more effective mimic the $3 \mathrm{D}$ architecture and treatment responses of cancer, as further discussed in the ESI. $\dagger$

Encouraged by the high uptake of AuSG-2Arg compared to AuSG in 2D cultures ${ }^{28}$ this study explored the therapeutic potential of both AuNC types as radiosensitizing agents for 
glioblastoma models. We report notable enhancement in radiotherapy efficacy by AuSG, and to a lesser extent by AuSG-2Arg, which was characterized by an increased necrotic response. The findings suggest that, despite a weak uptake, the AuSG are efficient radiosensitizers. In contrast, the AuSG-2Arg are weaker radiosensitizers than AuSG but more efficient diagnostic agents given their enhanced uptake. The reduced radiotherapy efficacy of AuSG-2Arg compared to AuSG may stem from the formation of extracellular aggregates (Fig. S6†), and its reduced capacity to augment ROS-induced oxidative stress (Fig. 4 and Fig. S11†). In addition, the AuSG appeared more effective to perfuse throughout the tumor compared to AuSG-2Arg (Fig. 2), potentially leading to a more homogeneous amplification of the radiotherapeutic effects throughout the microtumors by AuSG compared to AuSG-2Arg.

Mechanistically, our findings suggest that the AuNCs induce a "chemical radiotherapy enhancement" effect, ${ }^{29}$ which is further discussed in the ESI. $\dagger$ While the novel AuSG-Arg have strong potential for diagnostic imaging, their limited radiotherapeutic potential did not encourage further in vivo studies on radiotherapy enhancement with these nanoclusters. We demonstrate that the complex interactions of AuNC on cancer tissues and their theranostic potential can be effectively screened in 3D culture models, benefiting from the relative high-throughput of in vitro models while more faithfully recapitulating in vivo cancer tissues. The development of novel nanoclusters may thus benefit from the use of $3 \mathrm{D}$ cultures and emerging high-content assays for such models. ${ }^{2,30,31}$

\section{Conclusions}

Functionalization with arginine moieties greatly improves the uptake of AuNCs, enabling near- and shortwave infrared imaging with high sensitivity. Functionalization with glutathione is more suitable for radiotherapy enhancement, capable of achieving a 2-fold enhancement in overall efficacy. Our findings stress the importance of careful design and optimization of the surface chemistry of AuNCs for future radiotherapy applications. 3D cultures combined with highcontent imaging assays are highly suited to evaluate the theranostic properties of AuNCs, enabling accurate screening of promising particles for further in vivo studies.

\section{Conflicts of interest}

The authors have no conflicts to declare.

\section{Acknowledgements}

This work was funded by the Tumor Heterogeneity and Ecosystem program of Plan Cancer 2014-2019 (C16080CS). We additionally received support from Plan Cancer 20142019 (C18038CS), Cancéropôle Lyon Auvergne Rhône-Alpes, Fondation ARC (R17157CC), Région Rhône Alpes (Pack
Ambition Recherche 2017), Fondation pour la Recherche Médicale.

\section{Notes and references}

1 T. Kazda, A. Dziacky, P. Burkon, P. Pospisil, M. Slavik, Z. Rehak, R. Jancalek, P. Slampa, O. Slaby and R. Lakomy, Radiol. Oncol., 2018, 52, 121-128.

2 L. Zhang and E. Wang, Nano Today, 2014, 9, 132-157.

3 X. L. Guével, IEEE J. Sel. Top. Quantum Electron., 2014, 20, 45-56.

4 R. Jin, C. Zeng, M. Zhou and Y. Chen, Chem. Rev., 2016, 116, 10346-10413.

5 J. Xie, Y. Zheng and J. Y. Ying, J. Am. Chem. Soc., 2009, 131, 888-889.

6 D. Shen, M. Henry, V. Trouillet, C. Comby-Zerbino, F. Bertorelle, L. Sancey, R. Antoine, J.-L. Coll, V. Josserand and X. Le Guével, APL Mater., 2017, 5, 053404.

7 B. Musnier, K. D. Wegner, C. Comby-Zerbino, V. Trouillet, M. Jourdan, I. Häusler, R. Antoine, J.-L. Coll, U. ReschGenger and X. Le Guével, Nanoscale, 2019, 11, 1209212096.

8 Y. Chen, D. M. Montana, H. Wei, J. M. Cordero, M. Schneider, X. Le Guével, O. Chen, O. T. Bruns and M. G. Bawendi, Nano Lett., 2017, 17, 6330-6334.

9 J. Liu, M. Yu, C. Zhou, S. Yang, X. Ning and J. Zheng, J. Am. Chem. Soc., 2013, 135, 4978-4981.

10 C. Colombé, X. Le Guével, A. Martin-Serrano, M. Henry, E. Porret, C. Comby-Zerbino, R. Antoine, I. Atallah, B. Busser, J.-L. Coll, C. A. Righini and L. Sancey, Nanomedicine, 2019, 20, 102011.

11 J. H. Hubbell and S. M. Seltzer, X-Ray Mass Attenuation Coefficients, https:/www.nist.gov/pml/x-ray-mass-attenuation-coefficients, (accessed June 5, 2019).

12 K. T. Butterworth, S. J. McMahon, F. J. Currell and K. M. Prise, Nanoscale, 2012, 4, 4830-4838.

13 J.-L. Ravanat and T. Douki, Radiat. Phys. Chem., 2016, 128, 92-102.

14 M. Misawa and J. Takahashi, Nanomedicine, 2011, 7, 604-614. 15 J. D. Carter, N. N. Cheng, Y. Qu, G. D. Suarez and T. Guo, J. Phys. Chem. B, 2007, 111, 11622-11625.

16 J. F. Hainfeld, D. N. Slatkin and H. M. Smilowitz, Phys. Med. Biol., 2004, 49, N309-N315.

17 X.-D. Zhang, Z. Luo, J. Chen, X. Shen, S. Song, Y. Sun, S. Fan, F. Fan, D. T. Leong and J. Xie, Adv. Mater., 2014, 26, 4565-4568.

18 X.-D. Zhang, J. Chen, Z. Luo, D. Wu, X. Shen, S.-S. Song, Y.-M. Sun, P.-X. Liu, J. Zhao, S. Huo, S. Fan, F. Fan, X.-J. Liang and J. Xie, Adv. Healthcare Mater., 2014, 3, 133141.

19 X.-D. Zhang, Z. Luo, J. Chen, S. Song, X. Yuan, X. Shen, H. Wang, Y. Sun, K. Gao, L. Zhang, S. Fan, D. T. Leong, M. Guo and J. Xie, Sci. Rep., 2015, 5, 8669.

20 X. Le Guével, M. Henry, V. Motto-Ros, E. Longo, M. I. Montañez, F. Pelascini, O. de La Rochefoucauld, 
P. Zeitoun, J.-L. Coll, V. Josserand and L. Sancey, Nanoscale, 2018, 10, 18657-18664.

21 E. Porret, M. Jourdan, B. Gennaro, C. Comby-Zerbino, F. Bertorelle, V. Trouillet, X. Qiu, C. Zoukimian, D. Boturyn, N. Hildebrandt, R. Antoine, J.-L. Coll and X. Le Guével, J. Phys. Chem. C, 2019, 23, 26705-26717.

22 A.-L. Bulin, M. Broekgaarden and T. Hasan, Sci. Rep., 2017, 16445.

23 M. J. Reiniers, R. F. van Golen, S. Bonnet, M. Broekgaarden, T. M. van Gulik, M. R. Egmond and M. Heger, Anal. Chem., 2017, 89, 3853-3857.

24 M. Verheij and H. Bartelink, Cell Tissue Res., 2000, 301, 133-142.

25 E. Lechtman, N. Chattopadhyay, Z. Cai, S. Mashouf, R. Reilly and J. P. Pignol, Phys. Med. Biol., 2011, 56, 4631-4647.
26 N. N. Cheng, Z. Starkewolf, R. A. Davidson, A. Sharmah, C. Lee, J. Lien and T. Guo, J. Am. Chem. Soc., 2012, 134, 1950-1953.

27 M. Broekgaarden, R. Weijer, A. C. van Wijk, R. C. Cox, M. R. Egmond, R. Hoebe, T. M. van Gulik and M. Heger, J. Biomed. Nanotechnol., 2017, 13, 204-220.

28 E. Porret, J.-B. Fleury, L. Sancey, M. Pezet, J.-L. Coll and X. Le Guével, RSC Adv., 2020, 10, 6436-6443.

29 L. Cui, S. Her, G. R. Borst, R. G. Bristow, D. A. Jaffray and C. Allen, Radiother. Oncol., 2017, 124, 344-356.

30 M. Broekgaarden, A.-L. Bulin, J. Frederick, Z. Mai and T. Hasan, J. Clin. Med., 2019, 8, E1399.

31 M. Broekgaarden, A. Anbil, A.-L. Bulin, G. Obaid, Z. Mai, Y. Baglo, I. Rizvi and T. Hasan, Biomaterials, 1999, 222, 119421. 